\title{
Wind Turbine Tower Optimization under Various Requirements by Using Genetic Algorithm
}

\author{
Serdar Yıldırım, İbrahim Özkol \\ Faculty of Aeronautics and Astronautics, Istanbul Technical University, Istanbul, Turkey \\ E-mail: serdar.yildirim-2@postgrad.manchester.ac.uk,ozkol@itu.edu.tr \\ Received February 24, 2010; revised April 7, 2010; accepted April 11, 2010
}

\begin{abstract}
The purpose of this study is to optimize the mass of $1.5 \mathrm{MW}$ wind turbine steel tower performing Genetic Algorithm method (GA). In accordance with ASCE 7-98, AISC-89 and IEC61400-1, the impact of loads on tower is calculated within the highest safety conditions against buckling strength of each sections of tower by means of GA codes. The stifness along tower is ensured entirely while the mass of tower is mitigated and optimized.
\end{abstract}

Keywords: Mass Optimization, Genetic Algorithm, Wind Turbine Tower

\section{Introduction}

The cost of wind turbine towers can amount nearly $20-25 \%$ of the total investment cost for wind energy plant. Minimization of mass of wind turbine tower has become more crucial job for last two decades. Most modern wind turbines are installed with tubular conical steel towers from the aspect of aesthetics. They are ideally manufactured in 20-30 meters long welded sections and then bolted each other on site [1]. Steel tubular conical towers are manufactured as the tapered steel tower namely these towers have a conical shape with a wider base than the top in general. Such designs increase their strength and saves material. The tower of wind turbine gathers net loads from the tower head and transmits these loads to the foundation. The main load is the axial load on the rotor. Dynamic loading is generated by wind turbulence and constantly by blade tower interaction. The stiffness of tower is based on the tower top weight and the tower height. Additional design requirements have to be satisfied with adequate strength since admissible stresses are not exceeded and for conical towers, shell buckling must be prevented. The existing pre-sized tower is tackled to evaluate as per analysis and design conditions. Steel tower is assumed to be located in Balıkesir-Bandirma region in Turkey with $52 \mathrm{~m}$ tower height and the $54.7 \mathrm{~m}$ tower hub height. The top diameter of tower is $2.56 \mathrm{~m}$ and the base diameter tower is $4.3 \mathrm{~m}$.

\section{Allowable Stress Design (ASD)}

The steel towers are primarily designed and sized to meet the AISC strength design criteria. Allowable stress design method (ASD) is used in lieu of AISC-89 for the steel tubular tower design. The load combination method for the service load (characteristic load) condition is carried out with reference to ASCE-7-98. The allowable bending stress $F b$ for noncompact section is $0.6 F y$, in which the yielding stress $F y$ of the steel tubular structure is typically $345 \mathrm{MPa}$ (50 ksi). The allowable shear stress $F v$ is $0.4 F y$. The allowable compression stress $F a$ is represented by the following formula [2]:

$$
F a=\frac{\left[1-\frac{\left(K \frac{L}{r}\right)^{2}}{2 \cdot C_{c}^{2}}\right] \cdot F y}{\frac{5}{3}+\frac{3\left(K \frac{L}{r}\right)}{8 C_{c}}-\frac{\left(K \frac{L}{r}\right)^{3}}{8 C_{c}^{3}}}
$$

where $\left(K \frac{L}{r}\right)$ is the slenderness ratio of steel tower, $K$ $=2$ for the cantilever type of structure, and $L$ and $r$ are the length of the tower and radius of section, respectively. The material coefficient $C_{c}$ is calculated by:

$$
C_{c}=\sqrt{\frac{2 \cdot \pi^{2} \cdot E}{F y}}
$$


where $E$ is the steel modulus. When $K L / r$ is greater than $C_{c}$, the allowable compression stress $F a$ shall be recalculated by:

$$
F a=\frac{12 \cdot \pi^{2} \cdot E}{23 .\left(K \frac{L}{r}\right)^{2}}
$$

Typically, the ratio of the applied axial compression stress $f a$ to the allowable compression stress $F a$ of the steel tower is less than 0.15 . The combined stress for the applied bending stress $f b$ acting on the steel tower shall be satisfied with interaction equation.

$$
\frac{f a}{F a}+\frac{f b}{F b} \leq 1
$$

The applied shear stress $f v$ from the torsion and the shear force on the tower shall also be less than the allowable shear stress $F v$ [2].

\section{Fatigue Load}

The DEL (Damage Equivalent Load) method facilitates to determine the steel tower preliminary dimensions in any circumstances which fatigue load histogram data does not exist. The SN curve for the DEL method can be expressed in the following [2]:

$$
\log \log [\Delta \sigma s(n)]=\log \log (80 M P a)+\frac{2 \times 10^{6}-n}{m}
$$

The number of cycles corresponding to the withstand limit along the tower height $z$ can be calculated by using DEL method.

$$
N(z)=\frac{M f(z)}{\Delta \sigma_{r \max } S(z) \cdot \sqrt[m]{N_{0}}}
$$

where:

$M f(z)$ is the moment produced by the fatigue DEL thrust along steel tower. $S(z)$ is the section modulus that varies along the height of tower. $\Delta \sigma_{\text {rmax }}$ is the maximum allowable stress range at $N_{0}$ cycles (typically $\left.10^{4}\right) . m$ is the slope of the curve [2].

\subsection{Damage Equivalent Load for Steel Tower}

There are many fatigue calculation methods. One of which is DEL method in most cases where the full histogram of fatigue cycles is available but only a DEL specified. The DEL is added by a value of SNslope ( $m=4$ used in this circumstance and a number of cycles $(\mathrm{Ne})$ ).

Total moment range along the tower is calculated as follows:
$\Delta M x, y(z)=\frac{[\max \max (\Delta M x, y T)-\max \max (\Delta M x, y B)] z}{h}+$

$\max \max (\Delta M x, y B)$

$\max (\Delta M x, y T)=$ Maximum moment range at tower top $x$ or $y$ direction .

$\max (\Delta M x, y B)=$ Maximum moment range at tower base $\mathrm{x}$ or $\mathrm{y}$ direction.

Safety Factor of DEL is 1.0.

Consequence failure factor and material factor: $\gamma s d . \gamma m$ $=1.15 \times 1.1=1.265$

Number of cycles: $5.29 \times 10^{8}$ for $1.5 \mathrm{MW}$ turbine

This represents a 20 year lifetime.

\section{Local Buckling Stress}

The strength of the tubular steel tower in axial compression is the lesser of the yield strength and the elastic critical buckling stress $\sigma c r$ is calculated:

$$
\sigma_{c r}=0.605 E \cdot \frac{t}{r}
$$

where $r$ is the cylinder radius and $\mathrm{t}$ is the wall thickness. However, the presence of imperfections, particularly those introduced by welding, will significantly reduce the tower wall resistance to buckling. As per steel tower design, the reduction coefficient $\alpha_{0}$ for axial load is found by:

$$
\alpha_{0}=\left\{\begin{array}{lll}
\frac{0.83}{\sqrt{1+0.01 \cdot \frac{r}{t}}} \text { if } & \frac{r}{t}<212 \\
\frac{0.70}{\sqrt{0.1+0.01 \cdot \frac{r}{t}}} \text { if } & \frac{r}{t}>212
\end{array}\right.
$$

The reduction coefficient $\alpha B$ for bending load is calculated as follows:

$$
\alpha_{B}=0.1887+0.8113 \alpha_{o}
$$

The buckling stress $\sigma u$ can be computed in terms of the yielding stress $F y$ :

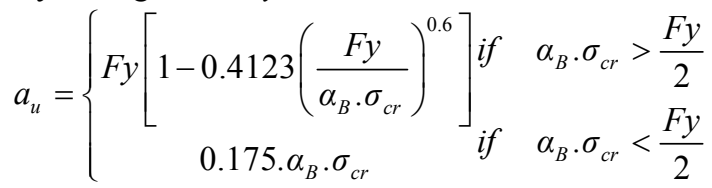

The maximum applied stress $\sigma a$ combined with normal stress and shear stress is calculated by $\sigma_{a}$ :

$$
\sigma_{a}=\sqrt{(f a+f b)^{2}+3 f v^{2}}
$$

The unity ratio for the combined stresses is found as follows. Now that the steel tower is liable to combined stress with axial compression and bending moment, the steel tower is designed to satisfy the combined stress check. This check named unity check interaction equa- 
tion is carried out in accordance with the AISC manual (ASD 9th Edition).

$$
\frac{f a}{F a}+\frac{f b}{F b} \leq 1 f b \leq 0.15 F b
$$

where:

$$
\begin{aligned}
& f a \quad \text { is the applied compression stress } \\
& F a \text { is the allowable stress } \\
& f b \quad \text { is the applied bending stress } \\
& F b \quad \text { is the allowable bending stress. }
\end{aligned}
$$

\section{Earthquake Load}

This section is based on ASCE 7-98 Earthquake Load Specification. Even though earthquake load seems to be not much significant effect on design of steel tower because of the fact that wind turbine towers are placed in low seismic areas, earthquake load should be taken into consideration so as to be more precise in designing of steel tubular tower (see Figure 1).

The maximum considered earthquake spectral response acceleration for short periods $\left(S_{M S}\right)$ and at 1 second $\left(S_{M 1}\right)$ adjusted for site class effects, should be determined by [3]:

$$
S_{M S}=F a . S s ; S_{M 1}=F v \cdot S_{1}
$$

where :

$S_{1}=$ Mapped maximum considered earthquake spectral response acceleration at a period of $1 \mathrm{~s}$ as determined in accordance with Section 9.4.1 (ASCE 7-89).

$S_{s}=$ Mapped maximum considered earthquake spectral response acceleration at short periods as determined in accordance with Section 9.4.1 (ASCE 7-89).

$F_{a}$ and $F_{v}$ are defined in Tables 9.4.1.2.4a and b respectively in accordance with Section 9.4.1 (ASCE 7-89). According to the ASCE 7-89 9.4.1.2.5 design spectral response acceleration at short periods, $S_{D S}$ and at $1 \mathrm{~s}$ period $S_{D 1}$ shall be determined from Equations 9.4.1.2.5-1 and 9.4.1.2.5-2 respectively:

$$
S_{D S}=\frac{2}{3} S_{M S} ; S_{D 1}=\frac{2}{3} S_{M 1}
$$

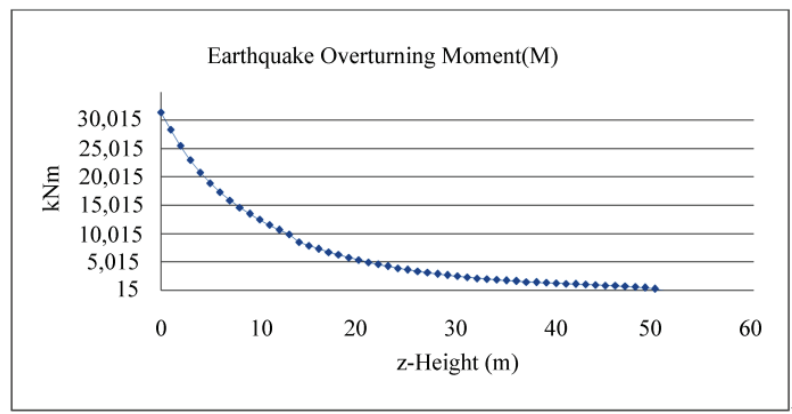

Figure 1. Earthquake overturning moment.
From the earthquake geographic map, the maximum considered earthquake (MCE) ground motion for soil site Category B with 5\% damping is $1.5 \mathrm{~g}(\mathrm{Ss})$ for structures with a short period of $0.2 \mathrm{~s}$ and $0.6 \mathrm{~g}(S 1)$ for structures with a period of $1 \mathrm{~s}$. The wind turbine towers are typically located in open areas away from population centers with very low occupancy. Because, the occupancy importance factor $(I)$ is equal to 1.0. Site Classification D is assumed for Balıkesir-Bandırma/ Marmara Region. Site Classification D is typified by stiff soils with shear velocity ( $V s$ in soil) typically $600-1,200 \mathrm{fps}(183-366 \mathrm{~m} / \mathrm{s})$.

$$
S_{D S}=\frac{2}{3} F_{s} S_{S}=1.0 g ; S_{D I}=\frac{2}{3} F_{v} S_{I}=0.6 g
$$

$F_{a}$ and $F_{v}$ can be defined according to the ASCE 7-98 Table 9.4.1.2.4a-4b respectively. $F_{a}$ is the site coefficient as a function of site class and short period MCE. $F_{v}$ is the site coefficient as a function of site class and a 1 second period MCEg is the acceleration caused by gravity.

$$
S_{a}(T)=\left\{\begin{array}{l}
\frac{S_{D I}}{T}, \text { if } T<T_{s} \\
S_{D S}\left(0.4+0.6 \frac{T}{T_{0}}\right), \text { if } \quad T<T_{0} \\
S_{D S} \text { Otherwise } \\
T_{S}=S_{D I} / S_{D S} ; T_{0}=0.2 . T_{s}
\end{array}\right.
$$

$T$ is the structural period.

Spectral acceleration response can be found as in Figure 2.

\subsection{Design Earthquake Load}

The earthquake lateral load affects the whole tower height $h$ as per its weight distribution [2].

$$
W=\int_{z}^{h} w(z) d z+W_{\text {Head Mass }}
$$

$w(z)$ is weight distribution as a function of height. $\mathrm{W}$ is the total weight of steel tower with Turbine Head. Base

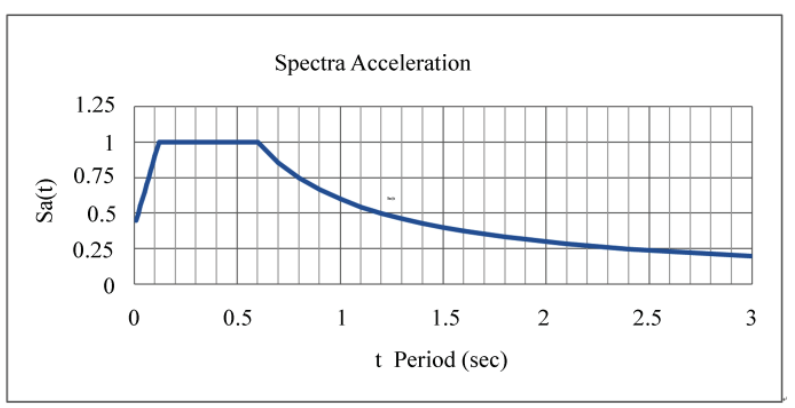

Figure 2. Spectra acceleration (earthquake). 
shear coefficient is,

$$
C_{s}(T)=S a(t) \cdot \frac{I}{R}
$$

$I$ is the importance factor. $R$ is the reduction factor equal to 1 .

Base Shear is $V=C_{s}(T) W$ (Figure 3)

The towers are assumed to be located in flat unobstructed area for direct wind exposure Category D where wind flows over the open water and flat terrain.

Importance factor is 1.0 for low occupancy concerning the wind turbine erection and installation.

\section{Wind Velocity Pressure}

The velocity pressure: (Figure 4)

$$
q_{z}=0.613 K_{z} K_{z t} K_{d} V^{2}\left(\frac{N}{m^{2}}\right)
$$

where:

The topographic factor $K_{z t}$ is 1.0 for the flat area.

$K_{d}$ is 0.95 for a round cylinder tower in accordance with Table 6-6 in ASCE 7-98.

The terrain exposure coefficient is determined as per Table 6-5 of ASCE 7-98 or by the following formula [3]:

$$
K_{z}(z)= \begin{cases}2.01\left(\frac{15 f t}{z_{g}}\right)^{\frac{2}{\alpha_{1}}} \text { ifz }<15 f t \\ 2.01\left(\frac{z}{z_{g}}\right)^{\frac{2}{\alpha_{1}}} \quad \text { otherwise }\end{cases}
$$

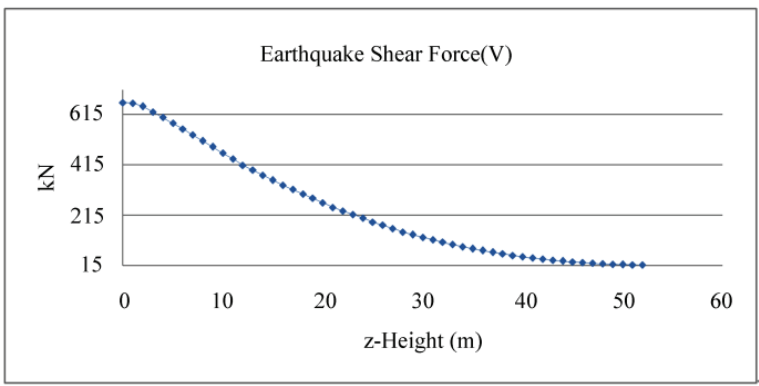

Figure 3. Earthquake shear force.

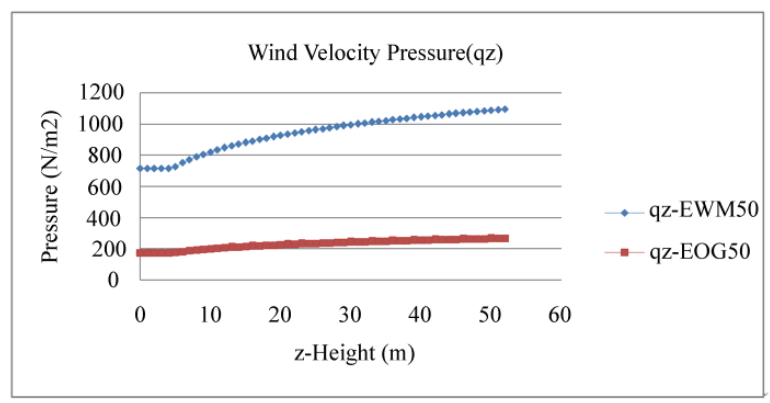

Figure 4. Wind velocity pressure on tower. where $z_{g}$ the nominal height of the atmospheric boundary layer is $213 \mathrm{~m}$ and $\alpha_{1}$ is 11.5 for exposure D category in accordance with ASCE 7-98.

\subsection{Direct Wind Load on Tower}

The direct wind load on the tower is not only based on the direct wind pressure on the tower but also on the gust factor $G_{f}$ and the force coefficient $C_{f}$.

$G_{f}$ is calculated by the following equation [2] :

$$
G_{f}=0.925\left(\frac{1+1.7 I_{z} \sqrt{g_{Q}^{2} Q^{2}+g_{R}^{2} R^{2}}}{1+1.7 g_{v} I_{z}}\right)
$$

where

The intensity factor of turbulence :

$$
I_{z}=0.15(33 \mathrm{ft} / z)^{1 / 6}
$$

The background response $Q$ and the resonant response are given in accordance with Eq.6.4 of ASCE 7-98.

$$
Q=\sqrt{\frac{1}{1+0.63\left(\frac{B+h}{L_{z}}\right)^{0.63}}}
$$

where $B$ is the horizontal dimension of tower measured normal to wind direction, $L_{z}$ is the integral length scale of the turbulence at the equivalent height given by $L_{z}=l(z / 33 f t)^{\epsilon} .1$ and $\epsilon$ are constants listed in Table 6.4 of ASCE 7-98.

$g_{R}$ and $g_{Q}$ shall be taken as the constant value of 3.4 and $g_{v}$ is given by:

$$
g_{v}=\sqrt{2 \ln \left(3600 n_{1}\right)}+\frac{0.577}{\sqrt{2 \ln \left(3600 n_{1}\right)}}
$$

$R$, the resonant response factor is given by:

$$
\begin{gathered}
R=\sqrt{\frac{1}{\beta} R_{n} R_{h} R_{B}\left(0.53+0.47 R_{L}\right)} \\
R_{n}=\frac{7.47 N_{1}}{\left(1+10.3 N_{1}\right)^{5 / 3}}
\end{gathered}
$$

The force coefficient $C_{f}$ is determined as per Tables 6-10 of ASCE 7-98. Lateral wind load along the tower is calculated by the direct pressure on the projected area which differs with respect to diameter distribution $d(z)$ of tower. $F_{z}(z)$ is determined in the following equation.

$$
\begin{array}{cc}
F_{z}(z)=q_{z} G_{f} C_{f} d(z) & (\text { Figure 5) } \\
V z(z)=\int_{\mathrm{z}}^{\mathrm{h}} F_{z}(x) d x & \text { (Figure 6) } \\
M z(z)=\int_{z}^{h} F_{z}(z) \cdot(x-z) d x & \text { (Figure 7) }
\end{array}
$$




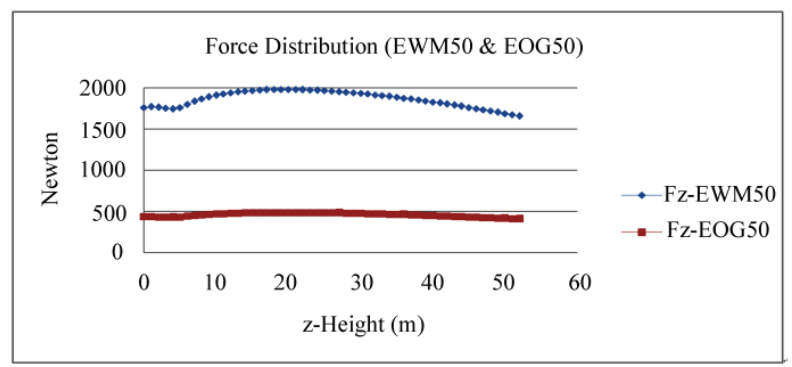

Figure 5. Force distributions because of direct wind effect on tower.

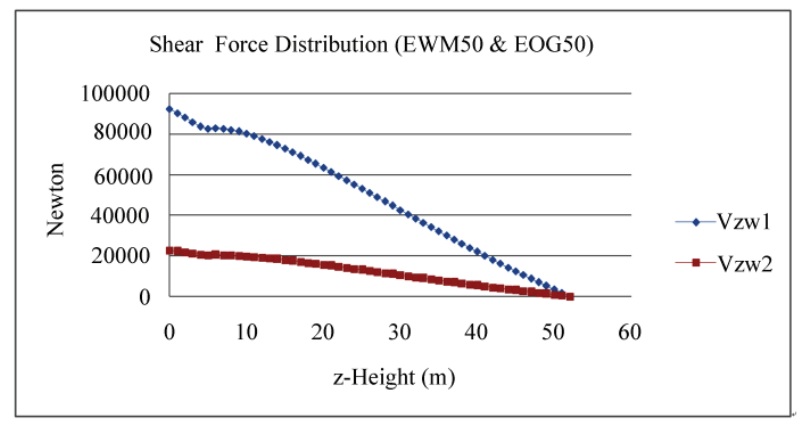

Figure 6. Wind shear force along tower.

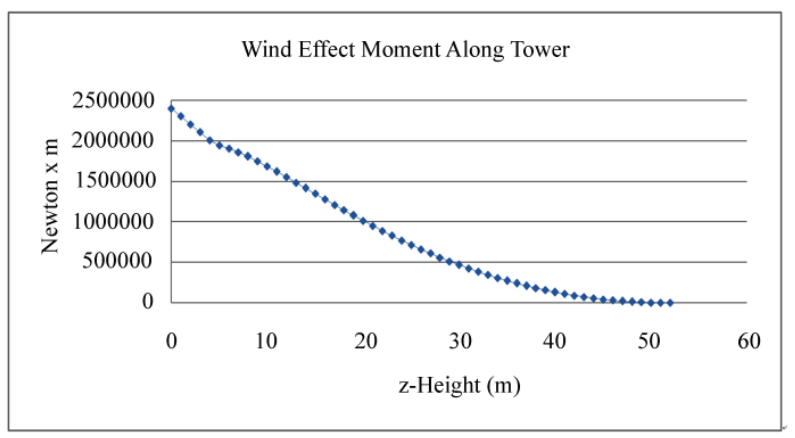

Figure 7. Wind effect moment along tower.

\section{Dynamic Behavior of Steel Tower}

Main key consideration in wind turbine design is the avoidance of resonant tower oscillations excited by rotor thrust fluctuations at rotational or blade passing frequency. Natural frequency of tower greater than the blade-passing frequency is said to be stiff on the other hand towers natural frequency between rotational frequency and blade passing frequency are regarded as soft. If the natural frequency, the tower is said to be soft-soft [4].

$$
w_{t}=1.75 \sqrt{\frac{E I g}{H^{3}\left(W_{t}+0.25 \text { Wtow }\right)}}
$$

where

$w_{t}$ is the estimated natural frequency of the tower is the height of tower. $E$ and $I$ are elastic modulus and moment of inertia of the tower. $W_{t}$ and Wtow are the weight of head mass and tower mass respectively.The natural frequency in $\mathrm{Hz}$ is calculated by: $f t=w_{t} / 2 \pi$

\section{Genetic Algorithm Approach}

Genetic Algorithms is one of the methods used in optimization problems. Particularly, it is based on natural selection. Genetic Algorithms is dependent on that the best generation has to live in nature. Although many genetic algorithms have been said with different structures, all of them comprises of three basic operations. Genetic Algorithm uses reproduction, crossover and mutation operators to define fitness and to create new solutions. Reproduction is simply a process to make decision which strings should remain and how many copies of them should be produced in the pool. The decision is made by comparing the fitness of each string. The fitness indicates survival potential and reproduction efficiency of the string in the next generations. For an Optimization problem, the fitness function is the objective function of optimization problem as shown in Figure 8.

\section{Optimization Problem}

Optimization problems are generally expressed as given in the following:

$p 1(x)$ : Margins of safety combined stress (bending stress and shear stress for torsion) because of the wind load effect.

$p 2(x)$ : Combined stress (bending stress and shear stress) because of the earthquake load effect.

$p 3(x)$ : Natural frequency for the 1st mode bending.

$p 4(x)$ : Fatigue stress.

Mtower: Mass of turbine tower.

Minimize

$f(x)=\operatorname{Mtower}(x) \cdot(1+p 1(x)+p 2(x)+p 3(x)+p 4(x))$

Constraints $12 \leq t_{x}^{i} \leq t_{x}^{i+1} \ldots . . \leq t_{x}^{i+51} \leq 26$ the thicknesses of sections

$$
i=1,2, \ldots \ldots \ldots \ldots \ldots \ldots \ldots ., 51
$$

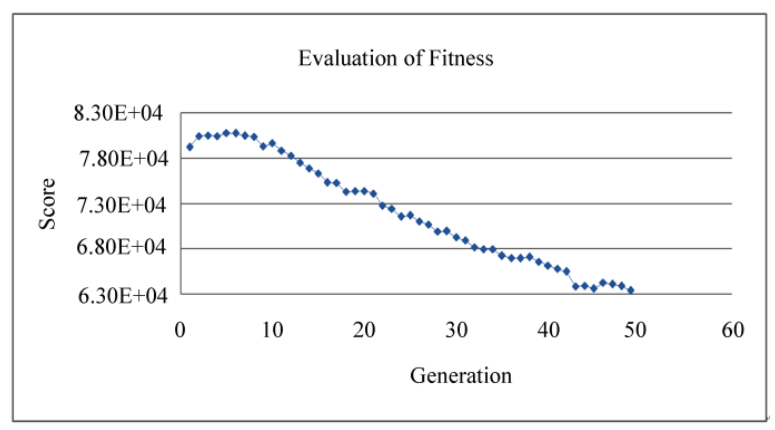

Figure 8. Evaluation of fitness function. 


\section{$U_{-} C_{-} W L(x) \leq D C R s w(x)$ (Figure 9).}

Unity check of critical combined buckling stress ratio due to wind effect load against combined stress ratio: $U_{-} C_{-} E Q(x) \leq D C R s q(x)$ (Figure 10).

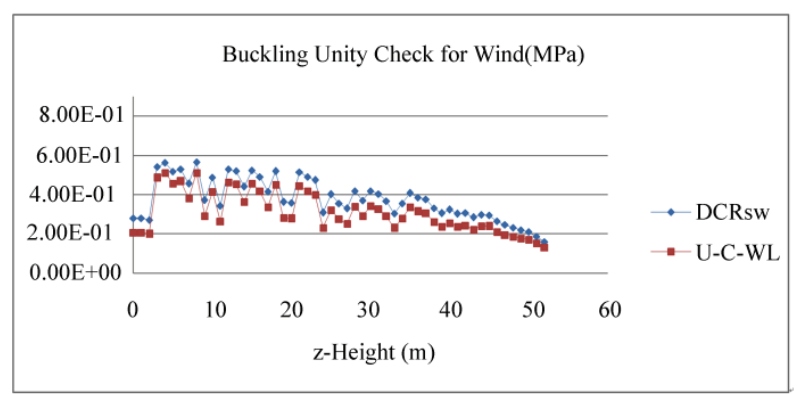

Figure 9. Buckling unity check for wind turbine effect and direct wind load.
Unity check of critical combined buckling stress ratio $U_{-} C_{-} E Q(x)$ due to earthquake effect load against combined stress ratio $\operatorname{DCRsq}(x) . w_{t}<w_{r}, w_{t}=$ Natural

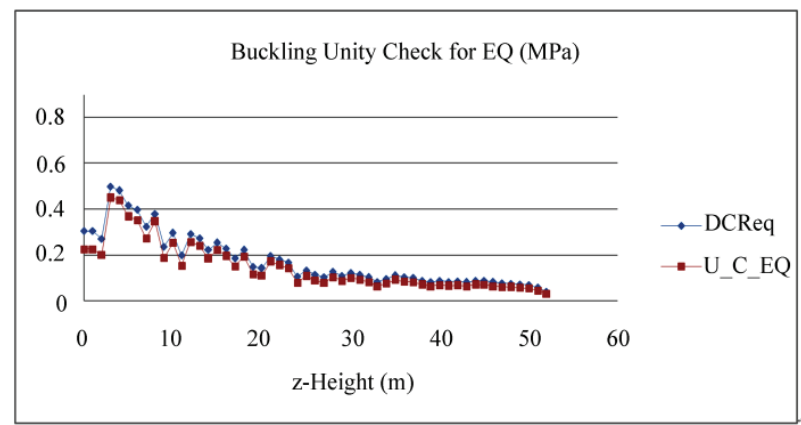

Figure 10. Buckling unity check for earthquake load (EQ).

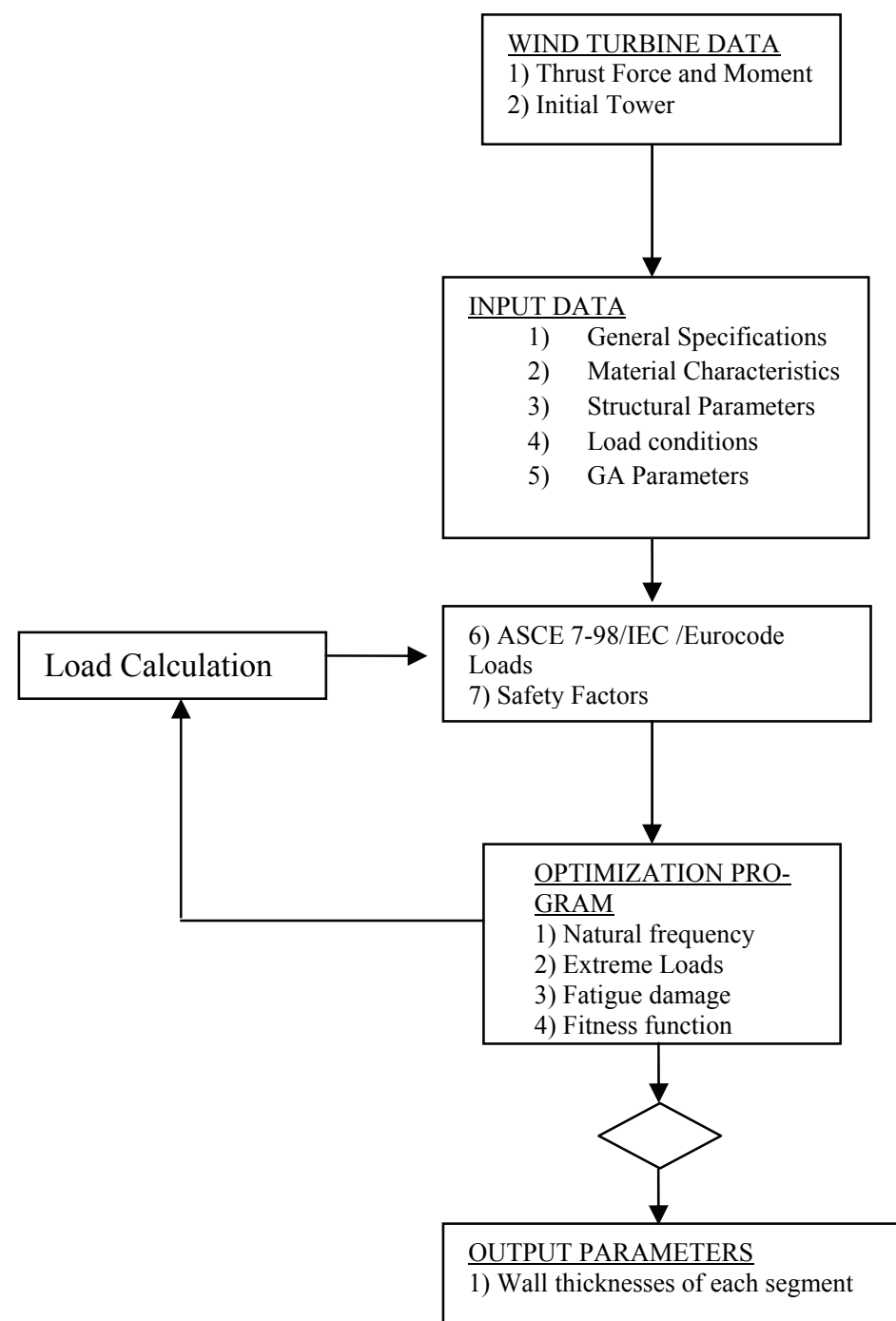

Figure 11. Design flow. 
frequency of tower1st mode $w_{r}=$ Operation frequency of turbine.

Design flow is shown as follows. This GA structure minimizes tower mass subject to general dimensions, design loads and some design restrictions. Load calculation depends on wind turbine design requirements of the standard IEC61400-1 and ASCE 7-98. All extreme loads of tower sections are calculated by the load combination [5] as shown in Figure 11.

\section{Optimization Results}

Score in figure above represents mass of steel tower in $\mathrm{kg}$. After $40^{\text {th }}$ generation of GA, value of mass levels out. In other words, optimization result has been reached at this point.

\section{Conclusions}

Each $1 \mathrm{~m}$ section along tower represented a chromosome in GA. Each section has been evaluated step by step on the basis of buckling strength in GA. An objective function has been produced by using a genetic algorithm. This optimizes the thickness of steel tower ranging from top $12 \mathrm{~mm}$ to base $26 \mathrm{~mm}$ in the distribution of pattern. All in all, the thicknesses of tower have been evaluated separately in each $1 \mathrm{~m}$ section along the height of the tower. Within the best solution of current conditions, the weight of tower has been obtained $63000 \mathrm{~kg}$ with a type of S355J0 material quality. Andit gives results for the best solution as indicated above in Figure 12. Moreover, the upcoming studies can be carried out and developed-

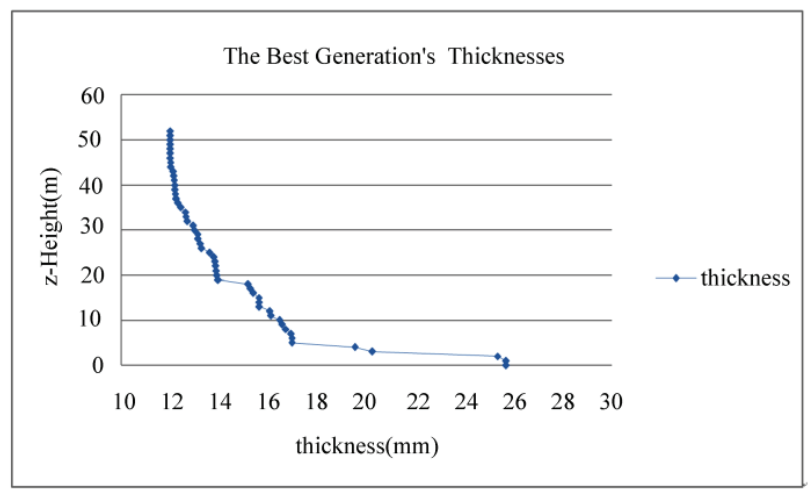

Figure 12. Thickness distribution.

further as longas stiffness is obtained.

\section{References}

[1] R. Y. Redlinger, P. D. Andersen and P. E. Morthorst, "Wind Energy in the 21st Century," Economics, Policy, Technology and the Cahnging Electricity Industry, 2002.

[2] "LWST Phase 1. Project Conceptual Design Study: Evaluation of Design and Construction Approaches for Economical Hybrid Steel/Concrete Wind Turbine Towers," 28 June 2002-31 July 2004, NREL Subcontractor Report, January 2005 NREL/SR-500-36777.

[3] ASCE 7-98, "Minimum Design Loads for Buildings and Other Structures," 1998.

[4] R. Harrison, E. Hau and H. Snel, "Large Wind Turbines," Design and Economics, 2000.

[5] IEC 61400-1, "Wind Turbine Generator System Part 1 Safety Requirements," 1999. 